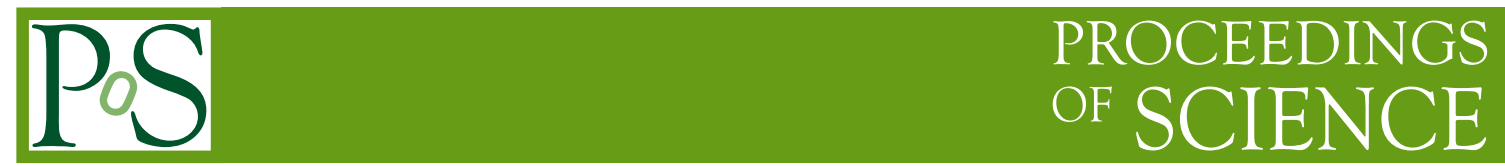

\title{
Rare electroweak penguin decays from Belle
}

\author{
HyoJung Hyun* \\ Kyungpook National University \\ E-mail: hjhyunegmail.com \\ (On behalf of the Belle Collaboration)
}

Electroweak penguin decays of $B$ mesons are sensitive probes of new physics beyond the Standard Model. We study the inclusive and exclusive electroweak penguin decays of $B$ meson. The measurements are based on a large data sample of $605 \mathrm{fb}^{-1}$ containing 657 millions $B \bar{B}$ pairs collected at the $\Upsilon(4 \mathrm{~S})$ with the Belle detector at the KEKB energy asymmetric $e^{+} e^{-}$collider.

The XIXth International Workshop on High Energy Physics and Quantum Field Theory, QFTHEP2010 September 08-15, 2010

Golitsyno, Moscow, Russia

${ }^{*}$ Speaker. 

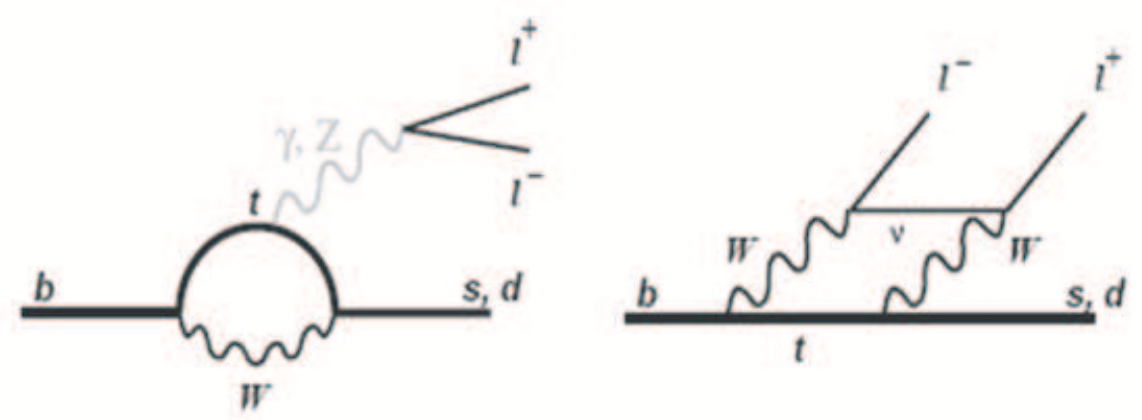

Figure 1: The penguin and box diagrams for the $b \rightarrow t \rightarrow(s, d)$ transition.

\section{Introduction}

Flavor changing neutral currents (FCNC) are forbidden at tree level in the Standard Model (SM) but are possible through loop-induced or higher order diagram. Figure 11 shows the penguin diagrams for the $b \rightarrow t \rightarrow(s, d)$ transition. Contributions from new physics (NP) [1] containing a charged Higgs boson or SUSY particles may arise in these electroweak loop processes. Therefore these transitions are sensitive to NP beyond the SM and provide a good opportunity to look for NP effects. In the effective Hamiltonian, Wilson coefficient is the strength of corresponding short distance operator. The effective Wilson coefficients $C_{7}, C_{9}$, and $C_{10}$ describe the amplitudes of the electromagnetic penguin, the vector electroweak, and the axial vector electroweak contributions, respectively [2]. These amplitudes may interfere with the contributions from non-SM particles [3]. Especially various measurement results of the $b$ to $s \ell^{+} \ell^{-}$decays such as the differential branching fraction (B.F.) and lepton forward-backward asymmetry $\left(A_{F B}\right)$ as a function of $q^{2}=M_{\ell \ell}^{2} c^{2}$ provide information on the Wilson coefficients associated with certain theoretical models [ []. In this paper, we present recent Belle results on various penguin decays.

\section{KEKB and Belle experiment}

All of these studies are based on $605 \mathrm{fb}^{-1}$ data sample which contains 657 million $B$ meson pairs collected at the $\Upsilon(4 S)$ resonance with the Belle detector [5] at the KEKB accelerator. The KEKB is an energy asymmetric $e^{+} e^{-}(3.5$ on $8 \mathrm{GeV})$ collider [6] operating at the energy of $\Upsilon(4 S)$ $(\sqrt{s}=10.58 \mathrm{GeV})$. The beams collide at a single interaction point (IP) with a crossing angle of \pm 11 mrad. The mass of the $\Upsilon(4 S)$ is just above the threshold of $B \bar{B}$ production and the $\Upsilon(4 S)$ decays exclusively to $B^{0} \overline{B^{0}}$ or $B^{+} B^{-}$.

The Belle detector is a large-solid-angle magnetic spectrometer consisting of a 5-layer silicon vertex detector (SVD), a 50-layer central drift chamber (CDC), an array of silica Aerogel threshold Čherenkov Counters (ACC), Time-of-Flight scintillation counters (ToF), and a CsI(TI) crystal electromagnetic calorimeter (ECL). These detectors are located inside a superconducting solenoid coil that provides a $1.5 \mathrm{~T}$ magnetic field. The SVD and CDC are used for vertexing/tracking and identification of charged particles. The ACC and ToF are used for charged particle identification. 
The ECL measures the energy of electromagnetic particles and is also used for electron identification. An iron flux-return located outside of the coil is instrumented to detect $K_{L}^{0}$ mesons and to identify muons.

To suppress large continuum background from $e^{+} e^{-} \rightarrow q \bar{q}$ events $(q=u, d, s, c)$, we use a selection criteria making use of the difference in the event topology between $B$ decays and continuum events. $B$ meson candidates are identified using the kinematic variables, the beam-energy constrained mass $M_{b c}=\sqrt{E_{b e a m}^{2}-p_{B}^{2}}$ and the difference between beam energy and $B$ meson $\Delta E=E_{B}-E_{\text {beam }}$, where $p_{B}$ and $E_{B}$ are the momentum and the energy of $B$ candidates in the center-of-mass frame, and $E_{\text {beam }}$ is the beam energy.

\section{3. $b$ to $s \ell^{+} \ell^{-}$}

\subsection{Exclusive $B \rightarrow K^{(*)} \ell^{+} \ell^{-}$study}

The signal events are reconstructed in 10 final states $K^{+} \pi^{-}, K_{S}^{0} \pi^{+}, K^{+} \pi^{0}, K^{+}$, and $K_{S}^{0}$ for $K^{(*)}$, combined with either electron or muon pairs. The particle selection criteria are described in detail elsewhere [2]. A $K^{(*)}$ candidate and a pair of oppositely charged leptons are combined to reconstruct $B$ meson candidates. The main backgrounds are continuum $e^{+} e^{-} \rightarrow q \bar{q}$ and semileptonic $B$ events. These backgrounds are suppressed by exploiting the differences between the signal and background. The dominant peaking backgrounds, $B \rightarrow J / \psi$ and $\psi^{\prime} X$ decays, are rejected explicitly in some of $q^{2}$ regions. The measured differential B.F. as a function of $q^{2}$ in $B \rightarrow K^{(*)} \ell^{+} \ell^{-}$are shown in Fig. 2 (a) and (b). The solid curves are the SM theoretical predictions with the minimum and maximum allowed form factors [7]. The two shaded regions are veto windows to reject the peaking backgrounds. The results are consistent with the SM prediction. The ratios of B.F. for the muon mode to the electron mode are calculated. The lepton flavor ratios, $R_{K}$ and $R_{K *}$, are sensitive to Higgs emission and the size of photon pole, respectively. The $R_{K}$ and $R_{K *}$ are predicted to be 1.0 and 0.75 in the SM. The results are measured to be $R_{K}=1.03 \pm 0.19 \pm 0.06$ and $R_{K *}=0.83$ $\pm 0.17 \pm 0.05$. They are consistent with the SM prediction. Angular variables are also sensitive to NP, and give information on the Wilson coefficients. The $K^{*}$ longitudinal polarization fraction $\left(F_{L}\right)$ and $A_{F B}$ are extracted from fits to $\cos \theta_{K *}$ and $\cos \theta_{B l}$, respectively. Figure 2 (c) and (d) show the $F_{L}$ and $A_{F B}$ as a function of $q^{2}$. In this figure, the solid curve is for SM prediction, and the dotted curve is for the opposite-sign $C_{7}$ model. The $F_{L}$ is consistent with the SM, but the $A_{F B}$ tends to be higher than the SM prediction. Isospin asymmetry $\left(A_{I}\right)$ is also measured as shown in Fig. 2 (e). The closed circle is for $K^{*} \ell \ell$ and the open circle is for $K \ell \ell$. As a result, there is no significant difference in $A_{I}$.

\subsection{Inclusive $B \rightarrow X_{S} \ell^{+} \ell^{-}$study}

For inclusive $B \rightarrow X_{s} \ell^{+} \ell^{-}$analysis with a semi-inclusive reconstruction technique, 36 exclusive decay modes are summed up. The $X_{s}$ hadronic system is reconstructed from one $K^{ \pm}$or $K_{S}^{0}$ and up to four pions, where at most one pion can be neutral. Possible 18 hadronic topologies are: $K^{ \pm}, K^{ \pm} \pi^{0}, K^{ \pm} \pi^{\mp}, K^{ \pm} \pi^{\mp} \pi^{0}, K^{ \pm} \pi^{\mp} \pi^{ \pm}, K^{ \pm} \pi^{\mp} \pi^{ \pm} \pi^{0}, K^{ \pm} \pi^{\mp} \pi^{ \pm} \pi^{\mp}, K^{ \pm} \pi^{\mp} \pi^{ \pm} \pi^{\mp} \pi^{0}$, $K^{ \pm} \pi^{\mp} \pi^{ \pm} \pi^{\mp} \pi^{ \pm}, K_{S}^{0}, K_{S}^{0} \pi^{0}, K_{S}^{0} \pi^{ \pm}, K_{S}^{0} \pi^{ \pm} \pi^{0}, K_{S}^{0} \pi^{ \pm} \pi^{\mp}, K_{S}^{0} \pi^{ \pm} \pi^{\mp} \pi^{0}, K_{S}^{0} \pi^{ \pm} \pi^{\mp} \pi^{\mp}, K_{S}^{0} \pi^{ \pm} \pi^{\mp} \pi^{\mp} \pi^{0}$, and $K_{S}^{0} \pi^{ \pm} \pi^{\mp} \pi^{\mp} \pi^{ \pm}$. According to the signal Monte Carlo simulation, the fraction of the $X_{S}$ decay 

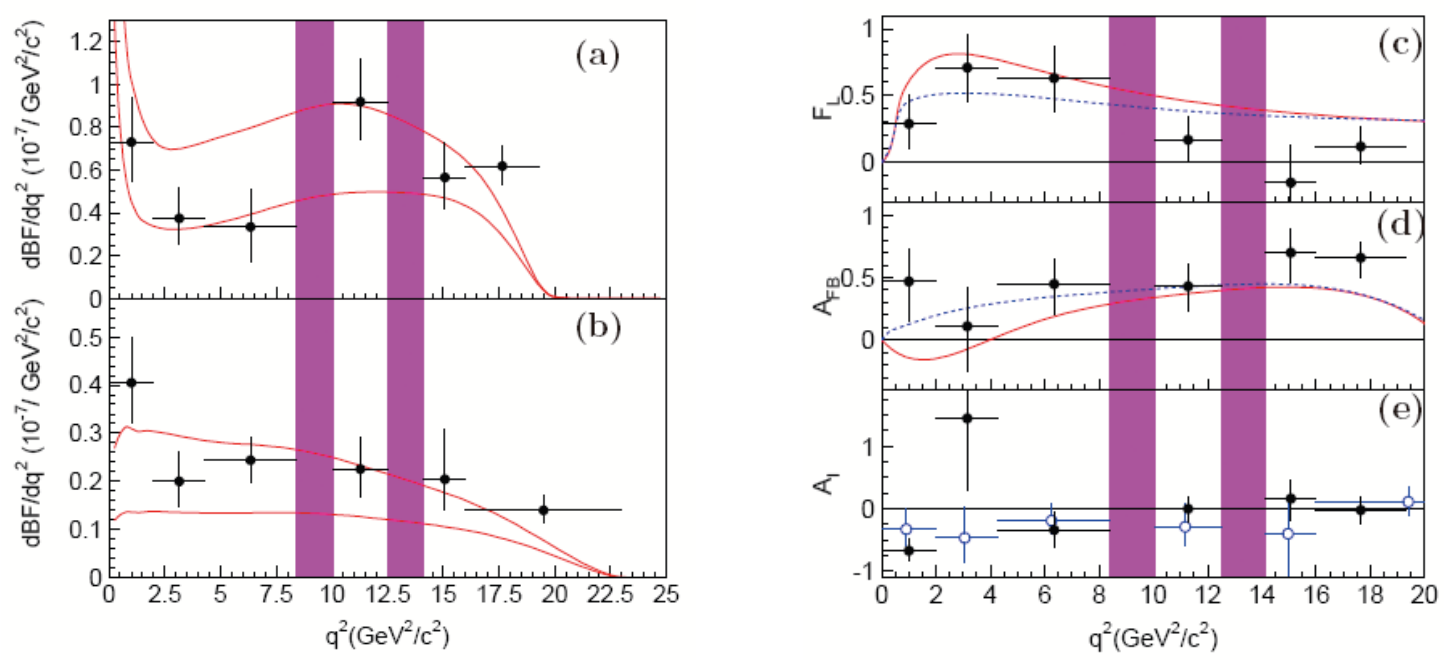

Figure 2: Differential branching fractions for the (a) $K^{*} \ell^{+} \ell^{-}$and (b) $K \ell^{+} \ell^{-}$modes as a function of $q^{2}$. (c) and (d) show the fit results for $F_{L}$ and $A_{F B}$ in $K^{*} \ell^{+} \ell^{-}$as a function of $q^{2}$ with the solid (dotted) curve representing the SM $\left(C_{7}=-C_{7}^{S M}\right)$ prediction [7]. (e) is the $A_{I}$ asymmetry as a function of $q^{2}$ for the $K^{*} \ell^{+} \ell^{-}$ (closed circle) and $\mathrm{K}^{+} \ell^{-}$(open circle) modes.

states covered by this semi-inclusive method is approximately $60 \%$. If the fraction of the states containing a $K_{L}^{0}$ is taken to be equal to that containing a $K_{S}^{0}$, the coverage is about $80 \%$.

The largest background source is random combinations from semileptonic $B$ decays. Each lepton to form the dilepton pair in $X_{s} \ell^{+} \ell^{-}$is picked up from decay products of different $B_{s}$, where $B$ decays into semileptonic state via the $b \rightarrow c \rightarrow s, d$ decay chain. This background has a significant amount of missing energy due to the neutrinos from the semileptonic decays. Another combinatorial background, which comes from continuum $q \bar{q}(q=u, d, s, c)$ background is also studied and rejected.

The inclusive B.F. is obtained by fitting the $M_{b c}$ distribution as shown in Fig. 3. In the Fig. 3, points with cross bar and red solid curve mean data and fit result, respectively. Black is signal component, yellow, green and blue are for backgrounds. Left plot is drawn and fitted when the $X_{s}$ mass is under $2.0 \mathrm{GeV} / c^{2}$ and for the right one, lower bound as like $1.0 \mathrm{GeV} / c^{2}$ for $X_{s}$ mass is added. Obtained significances are $10 \sigma$ and $3 \sigma$, respectively. The obtained B.F. is $\left(3.33 \pm 0.80_{-0.24}^{+0.19}\right) \times 10^{-6}$ for all the $X_{s}$ mass region with the above $0.2 \mathrm{GeV} / c^{2}$ in dilepton mass. This is consistent with the next and next leading order (NNLO) calculation in the SM.

\section{4. $b$ to $d \ell^{+} \ell^{-}$}

Events for the decays $B^{+} \rightarrow \pi^{+} \ell^{+} \ell^{-}$and $B^{0} \rightarrow \pi^{0} \ell^{+} \ell^{-}$, where $\ell^{+} \ell^{-}$is either a $\mu^{+} \mu^{-}$or $e^{+} e^{-}$ pair searched [8]. Signal events are reconstructed from a charged or a neutral pion candidate and a pair of oppositely charged electrons or muons. There is no significant signal and the obtained upper limit on the isospin-averaged B.F. at the $90 \%$ confidence level is $<6.2 \times 10^{-8}$. This is two times larger than the SM expectation. 

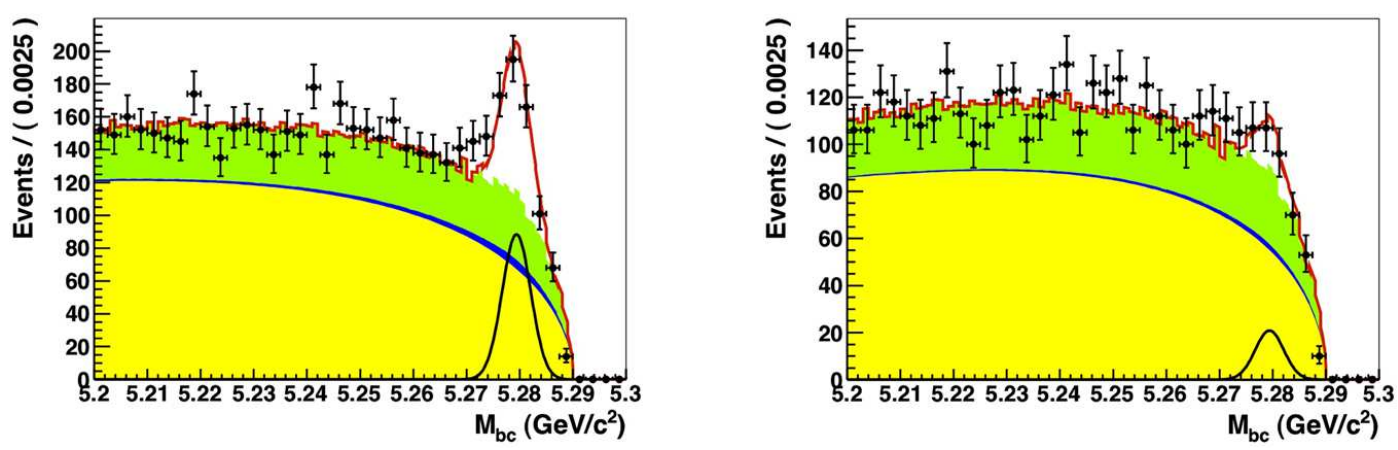

Figure 3: The $M_{b c}$ distribution fit result of the $605 \mathrm{fb}^{-1}$ data sample (Left: $M_{X_{s}}<2.0 \mathrm{GeV} / \mathrm{c}^{2}$, Right: 1.0 $\mathrm{GeV} / \mathrm{c}^{2}<M_{X_{s}}<2.0 \mathrm{GeV} / \mathrm{c}^{2}$ ). Background, peaking background, and self cross feed components are shown in yellow, green, and blue areas, respectively. Signal component is shown in black line.

\section{Summary}

We present the study results of exclusive and inclusive $b$ to $s, d$ semileptonic decays. Branching fraction, lepton flavor ratio $R_{K *}, F_{L}$, and $A_{I}$ for exclusive $B \rightarrow K^{*} l^{+} l^{-}$are consistent with SM prediction, but $A_{F B}$ measurement needs more statistics. Obtained branching fraction of inclusive $B \rightarrow X_{s} \ell^{+} \ell^{-}$is also consistent NNLO prediction in SM. The upper limit on the isospin-averaged branching fraction of exclusive $B \rightarrow \pi \ell^{+} \ell^{-}$is about twice the SM expectation. For this study, we need significantly more statistics and particle identification detector with better performance.

\section{References}

[1] A. Arhrib, C.-K. Chua and W.-W. Hou, Eur. Phys. J. C 21, 567 (2001); A. Ali and E. Lunghi, Eur. Phys. J. C 26, 195 (2002); Z.-J. Xiao and C. Zhuang, Eur. Phys. J. C 33, 349 (2004).

[2] J.-T. Wei et al., the Belle Collaboration, (2009) arXiv:0904.0771v1[hep-ex].

[3] S. Davidson, D. C. Bailey and B. A. Campbell, Z. Phys. C 61, 613 (1994); G. Burdman, Phys. Rev. D 52, 6400 (1995); J. L. Hewett and J. D. Wells, Phys. Rev. D 55, 5549 (1997).

[4] A. Ali, G. F. Giudice and T. Mannel, Z. Phys. C 67, 417 (1995); F. Kruger, L. M. Sehgal, N. Sinha and R. Sinha, Phys. Rev. D 61, 114028 (2000) [Erratum-ibid. D 63, 019901 (2001); A. Ali, E. Lunghi, C. Greub and G. Hiller, Phys. Rev. D 66, 034002 (2002); T. M. Aliev, A. Ozpineci and M. Savci, Eur. Phys. J. C 29, 265 (2003); and references therein. W. S. Hou, A. Hovhannisyan and N. Mahajan, Phys. Rev. D 77, 014016 (2008).

[5] A. Abashian et al., the Belle Collaboration, Nucl. Instrum. Meth. A479, 117 (2002).

[6] S. Kurokawa and E. Kikutani, Nucl. Instrum. Methods Phys. Res. Sect., A499, 1 (2003), and other papers included in this volume.

[7] A. Ali, P. Ball, L. T. Handoko and G. Hiller, Phys. Rev. D 61, 074024 (2000); A. Ali, E. Lunghi, C. Greub and G. Hiller, Phys. Rev. D 66, 034002 (2002).

[8] J.-T. Wei et al., the Belle Collaboration, Phys. Rev. D 78011101 (2008). 